\title{
Preguntones y creativos: discusiones y evidencias
}

\author{
Romina Cecilia Elisondo ${ }^{1}$ y Danilo Silvio Donolo ${ }^{2}$
}

\begin{abstract}
RESUMEN
La creatividad es una temática de interés científico, social y educativo. Cuestionar es una acción indispensable para la creatividad. En el artículo interesa presentar evidencias y discusiones respecto de las relaciones entre preguntas y creatividad, así como argumentar acerca de la pertinencia del procedimiento de evaluar las capacidades creativas a partir de la formulación de interrogantes. Analizamos estudios publicados en revistas internacionales en el período 2003-2016 en los que se utiliza el Test de Inteligencia Creativa CREA (Corbalán et al., 2003) como instrumento de medición. Las investigaciones indican relaciones entre el procedimiento utilizado por el CREA y los factores tradicionalmente asociados a la creatividad - fluidez, flexibilidad, originalidad y producción divergente- Asimismo, presentamos estudios que indican relaciones de baja intensidad entre creatividad, medida a partir de preguntas, e inteligencia. Mostramos investigaciones acerca de la estabilidad del procedimiento de formulación de preguntas ante diferentes estímulos y nos referimos a investigaciones que señalan relaciones entre logros y capacidades creativas. También recuperamos estudios sobre procedimientos alternativos de puntuación de preguntas en el CREA que consideran la originalidad de los interrogantes. Por último, argumentamos a partir de varios estudios respecto de las relaciones entre capacidades creativas, medidas mediante preguntas, y participación en diversas actividades de ocio. En las consideraciones finales analizamos las evidencias presentadas y destacamos los aportes del estudio al campo de la medición de la creatividad y los potenciales impactos en entornos educativos y cotidianos. Las preguntas son indicadores de capacidades creativas, tal como se sostiene en el CREA, y recursos valiosos para la promoción de procesos creativos en diversos contextos.
\end{abstract}

Palabras clave: creatividad, preguntas, test, originalidad, capacidades creativas, formulación de problemas.

\section{Questioners and Creatives: Discussions and Evidence}

\begin{abstract}
Creativity is a topic of scientific, social and educational interest. To question is an indispensable action for creativity. In the article, it is interesting to present evidences and discussions regarding the relations between questions and creativity, arguing about the pertinence of the procedure to evaluate creative capacities from the formulation of questions. We analyze studies published in international journals in the period 20032016, in which Test CREA (Corbalán et al., 2003) is used as a measuring instrument. Some studies present relationships between the procedure used by CREA and the factors traditionally associated with creativity -fluidity, flexibility, originality, divergent production-. We also present studies that indicate low intensity relationships between creativity, measured from questions, and intelligence. We show investigations about the stability of the process of formulating questions to different stimuli and researches that explore relationships between achievement and creative abilities. We also retrieve studies about an alternative score in the CREA that consider the originality of the questions. Finally, we argue from several studies about the relationships between creative abilities,
\end{abstract}

1 y 2 Consejo Nacional de Investigaciones Científicas y Técnicas. Conicet Universidad Nacional de Río Cuarto, Argentina; relisondo@gmail.com 
measures through questions, and participation in various leisure activities. In the final considerations, we analyze the evidence presented and highlight the contributions of the study to the field of measurement of creativity and the potential impacts on educational and everyday environments. The questions are related to creativity, are indicators of creative abilities, as it is maintained in the CREA, and resources for creative teaching and the promotion of creative processes in diverse contexts.

Keywords: creativity, questions, test, originality, creative abilities, problem formulation.

¿Por qué formular preguntas es un indicador de creatividad? Intentamos construir algunas respuestas a esta compleja pregunta a lo largo del escrito. Los estudios indican relaciones entre preguntas y creatividad (Aranguren, 2014; Corbalán \& Limiñana, 2010; Craft, 2013; Cremin et al., 2015; Elisondo \& Donolo, 2016a; Klimenko, Giraldo Arango, Dereix Restrepo, \& Montaño, 2014). Los creativos son preguntones, pueden formular múltiples y diversos interrogantes respecto de los objetos y las situaciones. Cuestionar parece ser una acción indispensable para la creatividad. En el artículo interesa presentar evidencias y discusiones respecto de las relaciones entre preguntas y creatividad, y argumentar acerca de la pertinencia del procedimiento de evaluar capacidades creativas a partir de la formulación de interrogantes.

La creatividad es una temática de interés científico, social y educativo. Existe cierto acuerdo respecto de la necesidad de crear contextos donde las personas pueden desarrollar procesos y productos creativos (Beghetto, 2017; Davies et al., 2013; Glaveanu, 2018). Organismos internacionales también han subrayado la relevancia de la creatividad como herramienta de innovación educativa y transformación cultural y sociali. En este marco, identificar personas y productos creativos resulta de interés en diferentes ámbitos laborales, educativos y cotidianos. A partir de los estudios iniciales de Guilford en la década de 1950, se han logrado significativos avances en el análisis de la creatividad y de los procedimientos para evaluar dicho constructo. Estos estudios iniciales se centraron en la evaluación del pensamiento divergente, es decir, de las posibilidades de generar múltiples ideas y soluciones ante determinadas situaciones. Originalidad, fluidez, flexibilidad y elaboración son los componentes principales del pensamiento divergente (Runco \& Acar, 2012). Desde mediados del siglo pasado hasta la actualidad se han desarrollado numerosos instrumentos de evaluación del pensamiento divergente. El Test de Pensamiento Creativo de Torrance (TTCT) es el instrumento más investigado y utilizado actualmente en diferentes lugares geográficos (Humble, Dixon, \& Mpofu, 2017; Primi, Nakano, Morais, Almeida, \& David, 2013; Yoon, 2017). En el contexto argentino se han realizado interesantes estudios psicométricos de dicho instrumento (Aranguren, 2014; Krumm, Aranguren, Arán Filippetti, \& Lemos, 2016).

En la actualidad, se han ido perfeccionado los instrumentos centrados en la valoración del pensamiento divergente $y$ se han construido nuevas herramientas de medición no solo del pensamiento divergente, sino también de otros aspectos y dimensiones de los procesos creativos (Batey, 2012; Park, Youngshin, \& Chun, 2016).

En el campo de investigación se han construido diferentes modelos para explicar la creatividad (Kozbelt, Beghetto, \& Runco, 2010), por ejemplo el de las $4 \mathrm{P}$ (persona, producto, proceso, press-entorno) y su versión ampliada que incluye $6 \mathrm{P}$ (persona, producto, proceso, entorno, persuasión y potencial). Este 
último modelo destaca la importancia de considerar en los procesos creativos las potencialidades de las ideas, los productos y las posibilidades de convencer a los demás respecto de la novedad y utilidad de estos. Teniendo en cuenta las clasificaciones de dichos modelos, en la presente investigación se consideran procesos creativos valorados por el Test CREA.

Kaufman y Beghetto (2009) proponen el modelo de las Cuatro C: creatividad con mayúsculas - transformaciones importantes en los campos de conocimientos-, creatividad con minúsculas - manifestaciones personales de la creatividad cotidiana-, mini-c creatividad -interpretaciones nuevas y personales de experiencias, acciones y eventos- y Pro-c creatividad creatividad que supone amplio nivel de conocimiento y experiencia en algún área específica, trasciende lo conocido como creatividad con minúsculas-. Los autores consideran que el modelo de las cuatro creatividades permite comprender los procesos creativos según su nivel de especificidad. Cada una de estas creatividades supone procedimientos diferentes de evaluación. La mini-creatividad es valorada mediante técnicas microgenéticas y de autoevaluación, la creatividad con minúsculas puede ser evaluada mediante test psicométricos, evaluaciones consensuales y valoraciones de pares, familiares y docentes, la pro creatividad a través de evaluación consensual, sistemas de citaciones, opiniones de especialistas y premios, la creatividad con mayúscula se identifica a partir de premios destacados y análisis historiométricos.

Los avances teóricos en el campo de la creatividad implicaron progresos en los instrumentos de medición y sus correspondientes procesos de validación. Las múltiples definiciones de creatividad y las diversas creatividades -creatividad con mayúsculas, creatividad con minúsculas, mini-c creatividad y pro-creatividad-demandan instrumentos y técnicas ajustados a dichos avances teóricos (Smith \& Smith, 2017). Si bien los test de pensamiento divergente son algunos de los más utilizados en el campo de investigación de la creatividad (Long, 2014; Runco, Abdulla, Paek, Al-Jasim, \& Alsuwaidi, 2016), se reconoce la existencia de diversas técnicas de evaluación de productos, logros y actividades creativas (Baer, Kaufman, \& Gentile, 2004; Conner \& Silvia, 2015; Kaufman, 2012; Silvia, Wigert, Reiter, \& Kaufman, 2012).

Evaluar la creatividad es un área de interés para los investigadores que se ha complejizado en función de los avances teóricos y metodológicos logrados en el campo. La diversificación de contextos de estudio de la creatividad también ha implicado cambios en los procedimientos de evaluación. Los avances de los estudios organizacionales y de las investigaciones de la creatividad cotidiana y educativa, también requieren técnicas específicas para cada contexto y área de conocimiento (Lubart \& Besançon, 2017).

El presente artículo trata sobre esta temática e intenta contribuir a la discusión respecto de técnicas y procedimientos para la valoración de la creatividad. Medir la creatividad es un desafio para los investigadores del campo de los procesos creativos. Se han desarrollado numerosos estudios y se lograron significativos avances, sin embargo aún quedan muchos interrogantes y dudas respeto de las propiedades técnicas y psicométricas de los instrumentos disponibles (Baer, 2011; Kim, 2011; Sternberg, 2012; Zeng, Proctor, \& Salvendy, 2011). 
El objetivo de este artículo es presentar evidencias empíricas respecto de la pertinencia de utilizar a las preguntas como indicador de capacidades creativas. Específicamente, se recuperan diversos estudios realizados por nuestro equipo y por otros investigadores en los que se estudia el Test CREA (Corbalán et al., 2003) como instrumento de evaluación de la creatividad. Se analizaron estudios publicados en revistas internacionales especializadas en el periodo 2003-2016. Los artículos analizados han sido publicados en las siguientes revistas especializadas: Anales de Psicología (Universidad de Murcia, España); RIES. Revista Iberoamericana de Educación Superior (Universidad Nacional Autónoma de México y Universia); RIDEP Revista Iberoamericana de Diagnóstico y Evaluación (Asociación Iberoamericana de Diagnóstico y Evaluación Psicológica); Boletín de Psicología (Universidad de Valencia) y Personality and Individual Differences (International Society for the Study of Individual Differences).

\section{¿Qué es la creatividad? ¿Cómo medirla?}

Son dos preguntas simples que inquietan a los investigadores de los procesos creativos. Estas dos preguntas se responden de diversas maneras conforme a los diferentes enfoques y procesamientos metodológicos desarrollados en el campo de la creatividad. Kozbelt, Beghetto y Runco (2010) identifican diez teorias que coexisten en el área de investigación de la creatividad; cada teoría se desarrolla según diferentes supuestos básicos, conceptos claves y focos de análisis. Las teorias psicométricas consideran que la creatividad puede ser medida a través de instrumentos estandarizados. Según las teorías del desarrollo, las personas despliegan la creatividad durante su vida a partir de las interacciones que se producen en diferentes contextos. Las teorias económicas desarrollan sus estudios desde la óptica del mercado y de los potenciales costos y beneficios de los procesos creativos. Las teorías componenciales analizan momentos y elementos de procesos creativos. Las teorias cognitivas investigan los procesos de pensamiento creativo combinación conceptual, expansión, metáforas-. Las teorias de resolución de problemas estudian las soluciones creativas y las teorias de formulación investigan los procedimientos de generación de problemas. Los procesos de evolución, generación y selección de ideas de creativos reconocidos son estudiados por las teorias evolucionistas. Las teorías tipológicas intentan identificar categorias de creadores. Y las teorías sistemáticas explican interrelaciones complejas entre diferentes elementos de los procesos.

Cada una de estas teorias ha cimentado definiciones acerca de la creatividad y los procesos creativos. Recuperamos algunos planteos que resultan relevantes para el objetivo de nuestro artículo. Interesa definir a la creatividad como un constructo complejo que incluye diferentes componentes y que se vincula con la originalidad, la divergencia, la fluidez y la flexibilidad del pensamiento $\mathrm{y}$ las acciones. Asimismo, se presentan algunas diferenciaciones necesarias para comprender este complejo constructo. Específicamente, se distingue entre potenciales y logros creativos, entre diferentes dimensiones o niveles de especificidad y entre diferentes focos de análisis - personas, productos, procesos y entornos-. También se plantean consideraciones respecto de la evaluación de la creatividad considerando las definiciones y diferenciaciones realizadas (Baer, 2012; Ivcevic, 2009; Lubart \& Besançon, 2017). 
La creatividad es un constructo que incluye diferentes componentes subjetivos y contextuales que se interrelacionan de manera compleja para la generación de pensamientos y productos novedosos y originales (Runco, 2010). Según Plucker, Beghetto y Dow (2004), las definiciones de creatividad incluyen conceptos referidos a la utilidad, la originalidad y el pensamiento divergente - fluidez, flexibilidad, originalidad y elaboración- Tales autores consideran que la creatividad se desarrolla en la interacción entre actitudes, procesos cognitivos y entornos; en dicha interacción se genera un producto novedoso y útil para determinado contexto social.

Definir a la creatividad como un constructo de dominio general o especifico también genera múltiples debates entre los investigadores de los procesos creativos (Baer, 2012; Barbot, Besancon, \& Lubart, 2016). Lógicamente, esta distinción también origina diferenciaciones entre los instrumentos. Mientras algunos intentan identificar un rasgo general vinculado a la creatividad, otros valoran diferentes áreas o dimensiones. En las teorias de la creatividad también es conveniente diferenciar entre potenciales y logros creativos; mientras los primeros refieren a capacidades y posibilidades de desarrollo de procesos creativos (Ivcevic, 2009), los segundos implican desempeños concretos en contextos y áreas particulares de conocimientos (Silvia et al., 2012). Las evaluaciones de potenciales creativos generalmente se realizan mediante un test de pensamiento divergente o resolución de tareas creativas (Amabile, 1996; Runco \& Acar, 2012). Según Lubart, Zenasni y Barbot (2013) los potenciales creativos deben medirse de diferente manera según la edad de los evaluados. En niños y adolescentes pueden utilizarse las tareas de pensamiento creativo; en cambio, en adultos las mediciones deben centrarse en los factores que subyacen a la creatividad y necesario realizar un conjunto medidas cognitivas y conativas para obtener un perfil creativo considerando dominios específicos. Para la evaluación de desempeños creativos, los instrumentos más utilizados son los cuestionarios y las escalas que valoran logros en diferentes áreas de conocimiento como por ejemplo, el Creative Behaviors Inventory (Hocevar, 1979), el Creative Achievement Questionnaire (Carson, Peterson, \& Higgins, 2005) y el Biographical Inventory of Creative Behaviors (Batey, 2007). En el contexto argentino también se han construido instrumentos de medición de comportamientos creativos (Aranguren \& Irrazabal, 2012) y acciones creativas (Elisondo \& Donolo, 2016b) en diferentes áreas de conocimiento.

Considerando nuestro objetivo en el presente artículo, interesa remarcar la idea de creatividad como potencialidad de formular ideas o cuestiones nuevas. Nuestros estudios se sustentan en las perspectivas de formulación y descubrimiento de problemas (Runco \& Okuda, 1988) y las que destacan la importancia de evaluar y promover potenciales creativos (Ivcevic, 2009). La publicación reciente de Smith y Smith (2017) enfatiza esta consideración, que propone una definición de creatividad centrada en las potencialidades creativas de las ideas. Según estos autores, la creatividad es el proceso de llegar con una buena idea, y una buena idea es aquella que tiene posibilidades de ser creativa. Como revisión de los criterios clásicos para identificar un producto creativo (utilidad, novedad, originalidad, valor, efectividad, etc.), Smith y Smith (2017) plantean una definición que denominan 1.5, donde el 1 representa la novedad de la idea y el .5 la potencialidad creativa de la misma. Los autores consideran necesario cambiar el enfoque centrado en la productividad inmediata de la idea hacia una perspectiva más amplia que 
considere las potencialidades de las mismas. Esta postura resulta relevante para pensar la creatividad en contexto educativo y la importancia de promover la formulación de ideas potencialmente creativas. En publicaciones recientes, Lubart y Besançon (2017) y Beghetto (2017) consideran relevante en el campo de la evaluación de la creatividad identificar potenciales creativos considerando particularidades según grupos etarios y contextos culturales.

En suma, múltiples debates caracterizan al campo actual de investigación de la creatividad y específicamente al área de la evaluación de capacidades, logros y procesos creativos. Para nuestras argumentaciones interesan especialmente los planteos que definen a la creatividad como una potencialidad general vinculada a la formulación de problemas. Estos planteos son precisamente los que fundamentan al test CREA como instrumento de medición de capacidades creativas (Corbalán et al., 2003).

\section{¿Qué propone el Test CREA?}

El Test de Inteligencia Creativa CREA (Corbalán et al., 2003) es un instrumento construido por un grupo de investigadores de la Universidad de Murcia (España) y de la Universidad Nacional de Río Cuarto (Argentina). Considerando las precisiones teóricas y metodológicas presentadas en el apartado anterior, el CREA es un instrumento psicométrico de medición de la creatividad que se sustenta en perspectivas vinculadas a la formulación de problemas. Asimismo, el CREA se apoya en teorias referidas a los factores clásicos de creatividad — producción divergente, flexibilidad, fluidez y originalidad-, al pensamiento lateral y a los estilos cognitivos (Corbalán \& Limiñana, 2010). El estilo psicológico creativo opera según una disposición general para la apertura y versatilidad de esquemas cognitivos; según los autores del CREA, la capacidad de las personas de formular preguntas es un indicador de dicha apertura a esquemas. Las preguntas surgen de la interacción entre el estímulo y la capacidad de las personas de abrirse a esa información y generar nuevos esquemas.

El objetivo del instrumento es evaluar capacidades creativas; en este sentido, se ubica dentro de las técnicas destinadas a valorar potenciales creativos. Por el contrario, el CREA no evalúa desempeños concretos y logros en campos particulares. Considerando los debates respeto de la creatividad como dominio general o especifico, el CREA es una medida indirecta y unitaria de capacidades creativas. Sin embargo, diversas investigaciones dan cuenta de correlaciones esta capacidad general y las dimensiones o factores tradicionales de la Batería de Guilford o el Test de Torrance (Clapham \& King, 2010; Corbalán et al., 2003; López Martínez \& Navarro Lozano, 2008).

Resulta tradicional en las pruebas de creatividad considerar distintos factores de ésta (...). El test que aquí se presenta propone, en cambio, una medida única de la creatividad. a la vista de los datos en los que se apoya, ésta parece quedar suficientemente justificada en cuanto a su capacidad para sintetizar y expresar la posición de un sujeto en una única variables que, sin embargo, resulta excelente predictora del conjunto de las de creatividad tradicionalmente definidas (Corbalán et al., 2003, p. 9).

El CREA ofrece una medida indirecta de la creatividad que utiliza como indicador a la capacidad de las personas de formular preguntas ante tres 
estímulos visuales (A, B y C). En este sentido, el TEST CREA permite la evaluación de la dimensión verbal de la creatividad. En la lámina A puede observase un teléfono antiguo; las láminas B y C representan situaciones extrañas o absurdas. Se trata de una prueba de ejecución máxima, la consigna general es: "Se te va a presentar una ilustración. Tu tarea consiste en escribir brevemente todas las preguntas que puedas hacer sobre lo que representa la lámina. Trata de hacer el mayor número posible de preguntas". Las personas disponen de cuatro minutos para realizar el mayor número posible de preguntas para cada lámina. El CREA es de aplicación individual para niños de 6 a 9 años. En niños mayores de 9 años, adolescentes y adultos la aplicación puede ser individual o colectiva. Se valora la cantidad de preguntas formuladas por las personas, se asignan puntajes adicionales para interrogantes que incluyen diferentes esquemas y contenidos. En el manual se presentan baremos para niños, adolescentes y adultos de población española $\mathrm{y}$ argentina, también se incluyen pautas que permiten clasificar a las producciones según tres criterios: creatividad alta, media o baja.

Numerosos estudios señalan las propiedades técnicas del instrumento y de los avances logrados en la medición de capacidades creativas mediante el procedimiento de la generación de interrogantes (Clapham \& King, 2010; Corbalán et al., 2003; 2014; López Martínez \& Brufau, 2010; López Martínez \& Navarro Lozano, 2008; Martínez Zaragoza, 2003). Los estudios psicométricos del instrumento se presentan de manera detallada en el manual (Corbalán et al., 2003). La fiabilidad se analizó mediante formas paralelas (.87). Se estudiaron relaciones entre el CREA y pruebas de Guilford para analizar la validez convergente, los resultados mostraron correlaciones significativas y moderadas entre los constructos $(r=.61 ; r=.75 ; r=.59 ; r=.48)$. En cambio, se observaron correlaciones bajas entre el CREA y pruebas de inteligencia $(r=$ $.25 ; r=.20$ ), lo que muestra la validez discriminante del instrumento. En el análisis de validez predictiva, se observaron correlaciones moderadas entre el CREA y puntuaciones de expertos respecto una tarea creativa (Corbalán et al., 2003). Los argumentos empíricos que se construyen a continuación también dan cuenta de las propiedades técnicas del CREA como instrumento de medición de la creatividad.

\section{¿Qué evidencias hay respecto de que las preguntas indican creatividad?}

Formular preguntas es un buen indicador para medir capacidades creativas. Esta afirmación se sustenta en perspectivas de la creatividad vinculadas a los estudios de procesos de formulación de problemas. Desde estas perspectivas se entiende que construir problemas es el procedimiento básico de la creatividad (Runco \& Okuda, 1988). Según Chen, Hu y Plucker (2016), la detección y creación de problemas es un elemento clave en el proceso creativo, generalmente un antecedente necesario para la producción creativa.

Formular preguntas, tal como considera Corbalán (2008), es en definitiva construir problemas. Plantear preguntas requiere de la activación de conocimientos y esquemas previos puestos en relación con nuevas informaciones. En el test CREA, cada nueva pregunta supone una relación inesperada entre el sujeto y el estímulo. No hay interrogantes correctos sino infinitas preguntas posibles, esta potencialidad del instrumento genera un contexto cognitivo propicio para el pensamiento divergente y la creatividad 
Interesa en este apartado presentar evidencias empíricas, resultados de estudios anteriores de nuestro equipo y de otros investigadores, donde se observa que la capacidad de formular preguntas se vincula con diferentes componentes, variables y procesos previamente asociados con la creatividad. En primer lugar, analizamos estudios que indican relaciones entre el procedimiento utilizado por el CREA y los factores tradicionalmente asociados a la creatividad (fluidez, flexibilidad, originalidad, producción divergente, etc.). En segundo lugar, presentamos estudios que indican relaciones de baja intensidad entre creatividad medida a partir de preguntas e inteligencia. En tercer lugar, mostramos investigaciones acerca de la estabilidad del procedimiento de formulación de preguntas ante diferentes estímulos. En cuarto lugar, nos referimos a investigaciones que indagan relaciones entre logros creativos y capacidades creativas. En quinto lugar, recuperamos estudios sobre procedimientos alternativos de puntuación de preguntas en el CREA que consideran la originalidad de los interrogantes. Por último, en sexto lugar, argumentamos a partir de varios estudios respecto de las relaciones entre capacidades creativas, medidas mediante preguntas, y participación en diversas actividades de ocio.

\section{Preguntas y factores de la creatividad}

Varios estudios dan cuenta de relaciones entre el procedimiento utilizado por el CREA y los factores tradicionalmente asociados con la creatividad. En la investigación realizada por Martínez Zaragoza (2003) se observaron correlaciones elevadas $(r=.75 ; r=.59 ; r=.78)$ entre las formas A, B y C del CREA y el factor de segundo orden subyacente a la Bateria de Creatividad de Guilford (1962). Los resultados del estudio de Clapham y King (2010) en población de habla inglesa demostraron interesantes correlaciones entre el Test CREA con el TTCT de Torrance (1990): dimensión verbal $(r=.50) \mathrm{y}$ dimensión figurativa $(r=.442)$. En tanto el CREA valora la dimensión verbal de la creatividad, se observan correlaciones más elevadas con esta dimensión del TTCT.

López Martínez y Navarro Lozano (2008) realizaron un análisis comparativo entre el TTCT de Torrance y el test CREA en una muestra de niños de la Región de Murcia (España). Los investigadores constataron asociaciones significativas entre las puntuaciones del CREA y tres de los factores del test de Torrance: fluidez, flexibilidad y originalidad. Asimismo, los datos muestran coincidencias al superponer las curvas de distribución de las puntuaciones CREA y de los factores fluidez o flexibilidad del TTCT. Según López Martínez y Navarro Lozano (2008), el estudio realizado aporta evidencias respecto de la validez concurrente del Test CREA.

En suma, los estudios citados en este apartado indican que formular muchas preguntas se relaciona con los factores de creatividad definidos por Guilford en la década de 1950. La ventaja del procedimiento del CREA es que se trata de un indicador unitario que resulta más simple y económico que las mediciones de factores como las que se utilizan en la Batería de Guilford y el Test de Torrance. Hacer preguntas parece ser una tarea que demanda pensamiento divergente, flexibilidad fluidez y originalidad.

\section{Preguntas e inteligencia}

Las teorias acerca de las relaciones entre creatividad e inteligencia son variadas. Sternberg y O`Hara (2005) diferencian cinco posturas al respecto: 
creatividad como elemento de la inteligencia; inteligencia como componente de la creatividad; creatividad e inteligencia como elementos, en algunos casos, similares o coincidentes y en otros diferentes, y creatividad e inteligencia como elementos coincidentes. Los autores del CREA (Corbalán et al., 2003) acuerdan con los planteos de Guilford respecto de la necesidad de un mínimo de inteligencia para la creatividad.

Los estudios de relaciones entre el CREA e instrumentos de medición de la inteligencia (Elisondo \& Donolo, 2010; Elisondo, Donolo, \& Corbalan, 2009) muestran resultados que se corresponden con los planteos de Guilford: los diagramas de dispersión indican que cierto mínimo de inteligencia es necesario para la creatividad; no se observan casos con puntajes elevados en las pruebas de creatividad y bajos en las de inteligencia. Las correlaciones observadas han sido bajas y no significativas. En los estudios de Martínez Zaragoza (2003) también se hallaron correlaciones muy bajas y no significativas entre el CREA y tres test de inteligencia (TEI Test Elemental de Inteligencia, OTIS Test de Inteligencia General y TEA Test de Aptitudes Escolares). Las correlaciones bajas entre el CREA y las mediciones de inteligencia también aportan al instrumento evidencias de validez discriminante.

Los estudios mencionados en el presente apartado demuestran que las personas que formulan muchas preguntas ante el CREA no son necesariamente las más inteligentes. El CREA no mide lo mismo que los instrumentos de medición de la inteligencia que se utilizaron en las investigaciones llevadas a cabo. Podriamos afirmar de manera provisoria que formular muchas preguntas no es un indicador de inteligencia, al menos de la inteligencia entendida como proceso convergente de resolución de problemas. Las relaciones entre creatividad e inteligencia han sido uno de los temas más investigados en el campo psicométrico de la creatividad; sin embargo, es uno de los tópicos respecto de los cuales menos acuerdos se han construido. Según Sternberg y O`Hara (2005), la mayoría de los investigadores avala la existencia de relaciones entre los constructos No obstante, la dirección de esas relaciones y las formas que adquieren en contextos reales y situaciones particulares son enigmas para el campo de la creatividad en particular y de la psicología en general.

\section{Preguntas más allá de los estímulos}

La capacidad de generar interrogantes es estable aun si se modifican los estímulos. Esta afirmación puede argumentarse considerando diversos estudios que demuestran correlaciones muy elevadas entre las tres láminas del CREA (Elisondo, 2015; Clapham \& King, 2010; Martínez Zaragoza, 2003).

Un estudio exploratorio (Elisondo \& Donolo, 2011) en el que incluimos nuevos estímulos (bolita de vidrio y sonidos de la naturaleza) también ofrece evidencias en este sentido. Se hallaron correlaciones elevadas entre los puntajes obtenidos por los participantes ante los diversos estímulos. Observamos que las personas formulan similar cantidad de preguntas ante los diferentes tipos de estimulos: visuales, auditivos y manipulativos.

Las capacidades de generar preguntas se mantienen estables aunque varien los estímulos y los formatos de los mismo (visuales, auditivos, etc.). Este dato es muy relevante para argumentar acerca de la pertinencia y estabilidad del procedimiento. Se ha observado que los resultados no se alteran modificando los estímulos. Formular preguntas parece ser una 
capacidad general que fácilmente se adapta a diferentes situaciones, contextos y estimulos. Es decir, la respuesta verbal que emerge de la aplicación del CREA no se ve afectada por el tipo de canal de entrada de información a la que se encuentre asociada el estímulo.

Cuestionar es una capacidad que no depende directamente de los objetos que operan como estímulos. Múltiples objetos y situaciones pueden activar variedad de preguntas y esquemas, lo que demuestra también la apertura y versatilidad que caracteriza a los procesos cognitivos vinculados a la creatividad.

\section{Preguntas entre la cantidad y la originalidad}

Recientemente realizamos dos estudios en los que analizamos las relaciones entre el procedimiento tradicional de puntuación del CREA, que considera principalmente la cantidad de preguntas formuladas, y un procedimiento alternativo, que atiende a la originalidad de las preguntas (Elisondo, 2015; Elisondo, Donolo, \& Limiñana, 2017). El sistema alternativo permite clasificar a las preguntas en muy frecuentes, frecuentes y únicas, y asigna puntajes diferenciales para cada tipologia. En las investigaciones se analizaron, según el procedimiento tradicional y el alternativo, preguntas formuladas por niños, adolescentes y adultos para las tres láminas del CREA. Los resultados indican correlaciones elevadas y significativas entre ambos sistemas en las tres láminas y en los tres grupos evaluados: niños, adolescentes y adultos. También se hallaron asociaciones significativas entre niveles de creatividad definidos con ambos sistemas. El análisis de casos indica que la gran mayoría de los participantes obtiene el mismo nivel de creatividad en los sistemas de puntuación. Sin embargo, se sugiere la utilización del sistema alternativo en casos de personas que formulan pocas preguntas, pero originales. En estos casos el sistema alternativo ofrece informaciones complementarias acerca de la originalidad de las producciones en el CREA.

Consideramos que estos resultados aportan evidencias respecto de la pertinencia del procedimiento utilizado por el CREA. En la mayoría de los casos, el procedimiento alternativo, más complejo y costoso, no aporta información adicional para la evaluación de la creatividad. Los análisis indican que valorar la cantidad de preguntas formuladas por las personas es un procedimiento parsimonioso y eficiente. Asimismo, evaluar la originalidad de las preguntas es una herramienta diagnóstica de relevancia en algunos casos donde la fluidez para el desarrollo de preguntas no está presente. En estos casos, es necesario valorar originalidad independientemente de fluidez. E1 aporte principal de los estudios realizados es haber analizado empíricamente, en muestras amplias y en las tres láminas, que el sistema tradicional resulta eficiente y económico en la mayoría de los casos (Elisondo, 2015; Elisondo, Donolo, \& Limiñana, 2017).

\section{La relación más consistente: preguntas y ocio}

En el campo e investigación de los procesos creativos se han presentado sobradas evidencias respecto de las relaciones entre apertura a la experiencia $\mathrm{y}$ creatividad. Los estudios indican que las personas más creativas son aquellas más abierta a las experiencias. La mayoria de los estudios que ofrecen estas afirmaciones las han realizado sobre la base de estudios correlacionales entre pruebas de creatividad y cuestionarios de personalidad 
(Chamorro-Premuzic \& Furnham, 2003; Hunter, Abraham, Hunter, Goldberg, \& Eastwood, 2016; Wolfradt \& Pretz, 2001).

En nuestros estudios (Chiecher, Elisondo, Paoloni, \& Donolo, 2018; Elisondo, 2013; Elisondo \& Donolo, 2014; 2015; 2016a) hemos observado relaciones entre creatividad y apertura a experiencias a partir de considerar los desempeños de las personas en contextos cotidianos. Específicamente, indagamos mediante cuestionarios respecto de actividades de tiempo libre y ocio activo. De manera consistente, en diferentes estudios y con diversos grupos, observamos que las personas que participan activamente en varias actividades de ocio obtienen en el CREA puntajes significativamente superiores respecto de quienes no participan. Este resultado es relevante en tanto indica que la capacidad de formular preguntas de alguna manera se vincula con la apertura a experiencias diversas de ocio. Las personas que más cuestionan parecen ser las más interesadas en vivenciar experiencias en contextos heterogéneos.

Son varias las evidencias que disponemos respecto de las relaciones entre las producciones en el CREA y la participación en actividades de ocio. En una investigación realizada con estudiantes de diferentes facultades de la Universidad Nacional de Río Cuarto $(n=549)$ observamos relaciones entre creatividad (utilizando el CREA) y participación en actividades extracurriculares. Los alumnos que obtienen puntajes más altos en el CREA son los que más participan en actividades extracurriculares. También entrevistamos a estudiantes que obtienen puntajes por encima del centil 90 en el CREA y observamos en ellos una disposición particular para participar activamente en contextos diversos dentro y fuera de la universidad (Elisondo \& Donolo, 2015).

Los datos de una investigación con estudiantes de Ingeniería de la Universidad Nacional de Río Cuarto $(n=132)$ también aportan evidencias a las relaciones entre el formular preguntas y el participar en actividades de ocio. Se ha corroborado la hipótesis que indica que los alumnos que participan en actividades de ocio serio obtienen puntajes significativamente superiores en las mediciones del CREA. También se ha observado que las personas que participan en más actividades de ocio obtienen mejores resultados en las puntaciones de creatividad (Chiecher et al., 2018).

Un estudio realizado con 300 personas adultas de diferentes niveles de escolaridad también demostró relaciones entre las producciones en el CREA y la participación activa en diversas propuestas de ocio. Se observó que las personas que participan en dos o más actividades de ocio activo obtuvieron puntajes significativamente superiores en el CREA respecto de quienes no participan. Las personas que participan en varias actividades al mismo tiempo logran los puntajes más elevados en el CREA (Elisondo, 2013).

También se observaron relaciones entre creatividad y participación en actividades de ocio en un estudio con 659 hombres y mujeres con diferentes nivel de escolaridad residentes en la provincia de Córdoba. Se observó que las personas que participan activamente en actividades de ocio estructuradas logran puntajes superiores en el test CREA y en otros instrumentos de medición de la creatividad respeto de quienes no participan. Asimismo se observó que los puntajes más elevados en el CREA los obtienen aquellas personas que participan en varias actividades de ocio (Elisondo \& Donolo, 2014). 
En un estudio sobre instrumentos de medición de la creatividad en un grupo amplio de 904 mujeres y hombres de diferentes edades y niveles de escolaridad se observaron en el CREA diferencias significativas entre grupos contrastados de personas que participan y no participan en actividades de ocio activo. Quienes participan obtienen en el CREA y en otros instrumentos de medición de la creatividad puntajes significativamente superiores respecto del resto (Elisondo \& Donolo, 2016a).

Los resultados que indican relaciones entre formular preguntas y participar en diversas actividades de ocio y tiempo libre también aportan evidencias respecto de la validez del procedimiento que utiliza el CREA. Participar en diversas actividades puede considerarse un indicador de apertura a la experiencia, es decir un interés especial por los entornos y las oportunidades que en ellos se ofrecen. Podría concluirse que las personas más cuestionadoras, las que formulan más preguntas en el CREA, son aquellas que se interesan por construir experiencias en diversos grupos y contextos. Cuestionar y participar parecen acciones interrelacionadas que indican de alguna manera creatividad y apertura a la experiencia como rasgos, estilos o constructos interrelacionados.

\section{CONSIDERACIONES FINALES}

Medir la creatividad, o las creatividades, fenómenos inasibles y complejos, no es una empresa sencilla para los investigadores. Claro está que, al igual que los demás constructos psicológicos, no es posible medir la creatividad como entidad, por lo que es necesario conformarse con algunos indicadores provisorios acerca de ciertas particularidades de los constructos. El Test CREA, de manera simple y económica, ofrece indicadores acerca de un proceso básico de la creatividad: formular problemas. El CREA no se ocupa de acciones o logros en diferentes contextos, sino del proceso que subyace a la producción creativa. Crear problemas o preguntas es el puntapié inicial del juego de la creatividad. Luego el partido se configura de diferentes maneras y culmina con resultados impredecibles

En el artículo presentamos evidencias empíricas, estudios previos que aportan a la discusión respecto del procedimiento de formular preguntas como indicador de creatividad. Las evidencias recuperan diferentes consideraciones teóricas y metodológicas del campo de la creatividad. Se aportaron evidencias sobre las complejas relaciones entre formular preguntas, los factores clásicos de la creatividad y la inteligencia. Asimismo, se presentaron datos que sustentan la validez del procedimiento independientemente de los estímulos y los sistemas de puntuación. Por último, a nuestro criterio, la evidencia más contundente refiere a las relaciones entre formular preguntas y participación en diversas actividades de ocio. Estas relaciones no son experimentales o artificiales, sino que se producen en contextos reales. Varios estudios dan cuenta de estas relaciones y ofrecen argumentos respecto de las interacciones entre cuestionar y participar, es decir entre creatividad y apertura a experiencias.

Además de los aportes al campo de la medición de la creatividad, las consideraciones realizadas en el presente artículo pueden tener impactos en la educación y la promoción de la creatividad en contextos cotidianos. Las preguntas como recursos educativos abren interesantes espacios en la enseñanza y el aprendizaje. Cada interrogante supone deseos de conocer y 
relaciones entre conocimientos previos y nuevos. Propiciar la formulación de preguntas por parte de los estudiantes es una estrategia didáctica propicia para la creatividad y el aprendizaje en diferentes campos de conocimiento. Preguntar es generar esquemas y abrirse a nuevos contenidos y objetos de la cultura (Elisondo \& Donolo, 2016b). Las perspectivas socioculturales de la educación enfatizan la importancia de las interacciones con personas y contenidos diversos (Rinaudo, 2014), las preguntas generan entornos favorables para aprender en diálogo con otros y con los objetos culturales. En un estudio reciente Gajda, Beghetto y Karwowski (2017) identificaron comportamientos de alumnos y docentes que promueven la creatividad en el aula. Según estos autores, plantear preguntas abiertas e incitar la formación de interrogantes son comportamientos que estimulan la creatividad en el aula. Galveanu y Beghetto (2017) también destacan la importancia de la apertura de diferentes perspectivas y preguntas en el contexto áulico para favorecer pensamientos creativos.

Las principales limitaciones del estudio se vinculan con las evidencias presentadas, la mayoría de ellas son investigaciones de nuestro equipo. En futuros estudios es necesario recuperar evidencias de otros investigadores y contextos de indagación a los efectos de ampliar y profundizar los análisis. Asimismo, en futuras investigaciones resulta relevante incorporar otras variables que ofrezcan evidencias respecto de las preguntas como indicadores de creatividad. Desarrollar estudios que aporten evidencias de validez considerando criterios externos y otros instrumentos de medición de la creatividad es indispensable para la mejora del Test CREA. Además, es necesario realizar estudios de confiabilidad mediante acuerdo de jueces, ya que los procedimientos de puntuación del CREA pueden verse afectados por interpretaciones diversas de sistema de correcciones propuesto en la prueba.

Considerar otros grupos y contextos, realizar estudios longitudinales de procesos creativos y atender a otros procedimientos de análisis de preguntas son futuras líneas de investigación promisorias de interés para el campo de la medición de la creatividad.

En suma, las preguntas se relacionan con la creatividad, son a la vez un indicador de capacidades creativas - tal como se sostiene en el CREA- y recursos para la enseñanza creativa. Mucho de la creatividad tiene que ver con cuestionar, indagar y buscar problemas y respuestas. Numerosos interrogantes quedan abiertos respecto de las relaciones entre la creatividad y preguntas, interrogantes que indican posibilidades de más creatividad, aprendizajes y conocimientos.

i En los siguientes documentos de la UNESCO se observa que la creatividad es un temática prioritaria que atraviesa diversas áreas de acción:

Nuestra diversidad creativa http://unesdoc.unesco.org/images/0010/001055/105586sb.pdf

Politicas para la creatividad https://es.unesco.org/creativity/sites/creativity/files/220384s.pdf;

Igualdad de género. Patrimonio y Creatividad http://unesdoc.unesco.org/images/0023/002316/231661s.pdf

Diversidad de las expresiones culturales http://es.unesco.org/creativity/convenci\%C3\%B3n/qu\%C $3 \% \mathrm{~A} 9$-es/texto

\section{REFERENCIAS}

Amabile, T. (1996). Creativity in context. Boulder, CO: Westview Press

Aranguren, M. (2014). Validez de constructo del Test de Pensamiento Creativo de Torrance en una muestra de jóvenes argentinos. Anuario de Psicología, 44 (1), 55-70.

Aranguren, M. \& Irrazabal, N. (2012). Diseño de una escala para la evaluación del comportamiento creativo en diferentes dominios. Ciencias Psicológicas, 4, 29-41.

Baer, J. (2011). How divergent thinking tests mislead us: Are the Torrance tests still relevant in the $21 \mathrm{st}$ 
century? The Division 10 debate. Psychology of Aesthetics, Creativity, and the Arts, 5(4), 309-313. https://doi.org/10.1037/a0025210

Baer, J. (2012). Domain specificity and the limits of creativity theory. The Journal of Creative Behavior, 46(1), 16-29. https://doi.org/10.1002/jocb.002

Baer, J., Kaufman, J., \& Gentile, C. (2004). Extension of the Consensual Assessment Technique to Nonparallel Creative Products. Creativity Research Journal, 16(1), 113-117. https://doi.org/10.1207/s15326934crj1601_11

Barbot, B., Besancon, M., \& Lubart, T. (2016). The generality-specificity of creativity: Exploring the structure of creative potential with EPoC. Learning and Individual Differences, 52, 178-187. https://doi.org/10.1016/j.lindif.2016.06.005

Batey, M. (2007). A psychometric investigation of everyday creativity. Unpublished Doctoral Thesis. University College, London.

Batey, M. (2012). The measurement of creativity, from definitional consensus to the introduction of a new heuristic framework. Creativity Research Journal, 24(1), 55-65. https://doi.org/10.1080/10400419.2012.649181

Beghetto, R. (2017). CODA: Creative Contradictions: Common Themes, Unique Insights, and Future Directions. (pp. 349-355). En R. Beghetto \& B. Sriraman (Eds.), Creative Contradictions in Education. Cham: Springer International Publishing. https://doi.org/10.1007/978-3-319-21924-0

Carson, S., Peterson, J., \& Higgins, D. (2005). Reliability, validity, and factor structure of the creative achievement questionnaire. Creativity Research Journal, 17(1), 37-50. https://doi.org/10.1207/s15326934crj1701_4

Chamorro-Premuzic, T. \& Furnham, A. (2003). Personality predicts academic performance: Evidence from two longitudinal university samples. Journal of research in personality, 37(4), 319-338. https://doi.org/10.1016/S0092-6566(02)00578-0

Chen, B., Hu, W., \& Plucker, J. A. (2016). The effect of mood on problem finding in scientific creativity. The Journal of Creative Behavior, 50(4), 308-320. https://doi.org/10.1002/jocb.79

Chiecher, A., Elisondo, R., Paoloni, P., \& Donolo, D. (2018). Creatividad, género y rendimiento académico en ingresantes de ingeniería. Revista Iberoamericana de Educación Superior, 9(24), 138-151.

Clapham, M. \& King, W. (2010). Psychometric characteristics of the CREA in an english speaking population. Anales de Psicología, 26(2), 206-211.

Conner, T. \& Silvia, P. (2015). Creative days: A daily diary study of emotion, personality, and everyday creativity. Psychology of Aesthetics, Creativity, and the Arts, 9(4), 463-470. https://doi.org/10.1037/aca0000022

Corbalán, J. (2008). ¿¿De qué se habla cuando hablamos de creatividad? Cuadernos FHyCSUNJu, 35, 11-21.

Corbalán, J. \& Limiñana, M. (2010). El genio en una botella. El test CREA, las preguntas y la creatividad. Anales de Psicología, 26(2), 197-205.

Corbalán, J., Martin-Brufau, R., Donolo, D., Clapham, M., Limiñana, R., García Peñas, V., \& King, R. (2014). CREA.A cross-cultural study. Personality and Individual Differences, 60, S54. https://doi.org/10.1016/j.paid.2013.07.223

Corbalán, J., Martinez, F., Autor, D., Alonso, C., Tejerina, M. y Limiñana, M. (2003). CREA. Inteligencia Creativa. Una medida cognitiva de la creatividad. Madrid: TEA Ediciones.

Craft, A. (2013). Childhood, possibility thinking and wise, humanizing educational futures. International Journal of Educational Research, 61, 126-134. https://doi.org/10.1016/j.ijer.2013.02.005

Cremin, T., Glauert, E., Craft, A., Compton, A., \& Styliandou, F. (2015). Creative little scientists: exploring pedagogical synergies between inquiry-based and creative approaches in Early Years science. Education, 43(4), 404-419. https://doi.org/10.1080/03004279.2015.1020655

Davies, D., Jindal-Snape, D., Collier, C., Digby, R., Hay, P., \& Howe, A. (2013). Creative learning environments in education A systematic literature review. Thinking Skills and Creativity, 8, 80-91. https://doi.org/10.1016/j.tsc.2012.07.004

Elisondo, R. (2013). Potencialidades creativas en contextos cotidianos. En D. Donolo \& R. Elisondo (Eds.) Estudio de Creatividad. Las travesias de Alfonsina, de Astor, de Julios y de Marias (pp. 47-390). Tenerife: Sociedad Latinoamericana de Comunicación Social.

Elisondo, R. (2015). Evaluación de la creatividad. Análisis de variables alternativas relacionadas con la forma y el contenido de las respuestas en el CREA. Tesis de Doctorado. Universidad de Murcia.

Elisondo, R. \& Donolo, D. (2010). Creatividad o inteligencia. This is not the question. Anales de Psicología, 26(2), 220-225.

Elisondo, R. \& Donolo, D. (2011). Los estímulos en un test de Creatividad. Incidencias según género, edad y escolaridad. Boletin de Psicología, 101, 51-65.

Elisondo, R. \& Donolo, D. (2014). Interculturalidad, apertura a experiencias y creatividad Aportes para una educación alternativa. Revista Red, 41, 5-19.

Elisondo, R. \& Donolo, D. (2015) Creatividad en la Universidad. Cuadernos de Educación. Tenerife: Sociedad Latina de Comunicación Social.

Elisondo, R. \& Donolo, D. (2016a) Creativity, Involvement, and Leisure Time: The More, the Better. Business creativity and the creative economy, 2(1), 102-109.

Elisondo, R. \& Donolo, D. (2016b). Construcción y análisis de las propiedades psicométricas del Cuestionario de Acciones Creativas en población argentina. PSIENCIA. Revista Latinoamericana de Ciencia Psicológica, 8(1), 1-21.

Elisondo, R., Donolo, D., \& Corbalán, J. (2009). Evaluación de la Creatividad ¿¿Relaciones con inteligencia y personalidad? Revista Iberoamericana de Diagnóstico y Evaluación Psicológica, 28(2), 67-79.

Elisondo, R., Donolo, D. S., \& Limiñana, R. (2017). The measure of originality in CREA test responses. Anales de Psicologia/Annals of Psychology, 34(1), 197-210. 
https://doi.org/10.6018/analesps.34.1.286131

Gajda, A., Beghetto, R., \& Karwowski, M. (2017). Exploring creative learning in the classroom: A multimethod approach. Thinking Skills and Creativity, 24, 250-267. https://doi.org/10.1016/j.tsc.2017.04.002

Glaveanu, V. (2018). Educating which creativity? Thinking Skills and Creativity, 27, $25-32$. https://doi.org/10.1016/j.tsc.2017.11.006

Glaveanu, V. \& Beghetto, R. (2017). The Difference That Makes a 'Creative' Difference in Education. (pp. 3754). En R. Beghetto, R. \& B. Sriraman (Eds.), Creative Contradictions in Education. Cham: Springer International Publishing.

Guilford, J. P. (1962). Creativity: it's measurement and development. En S. Parnes \& H. Harding (Eds.), A source book for creative thinking. New York: Scribner's.

Hocevar, D. (abril, 1979). The development of the Creative Behavior Inventory (CBI). Paper presented at the annual meeting of the Rocky Mountain Psychological Association.

Humble, S., Dixon, P., \& Mpofu, E. (2017). Factor Structure of the Torrance Tests of Creative Thinking Figural Form A in Kiswahili speaking children: multidimensionality and influences on creative behavior. Thinking Skills and Creativity, 27, 33-44. https://doi.org/10.1016/j.tsc.2017.11.005

Hunter, J., Abraham, E., Hunter, A., Goldberg, L., \& Eastwood, J. (2016). Personality and boredom proneness in the prediction of creativity and curiosity. Thinking Skills and Creativity, 22, 48-57. https://doi.org/10.1016/j.tsc.2016.08.002

Ivcevic, Z. (2009). Creativity map: Toward the next generation of theories of creativity. Psychology of Aesthetics, Creativity, and the Arts, 3, 17-21. https://doi.org/10.1037/a0014918

Kaufman, J. C. (2012). Counting the muses: Development of the Kaufman Domains of Creativity Scale (KDOCS). Psychology of Aesthetics, Creativity, and the Arts, 6(4), 298-315. https://doi.org/10.1037/a0029751

Kaufman, J. \& Beghetto, R. (2009). Beyond big and little, the four C model of creativity. Review of General Psychology, 13, 1-12. https://doi.org/10.1037/a0013688

Kim, K. (2011). The APA 2009 Division 10 debate. Are the Torrance Tests of creative thinking still relevant in the 21 st century? Psychology of Aesthetics, Creativity and the Arts, 5(4), 302-308. https://doi.org/10.1037/a0021917

Klimenko, O., Giraldo Arango, P., Dereix Restrepo, A., \& Montaño, W. (2014) Fomento del componente cognitivo de la capacidad creativa mediante la metodología de formulación de preguntas investigativas. Psicoespacios, 8 (2), 378-401.

Kozbelt, A., Begheto, R. \& Runco, M. (2010). Theories of Creativity. En J. Kaufman \& R. Sternberg (Eds.), The Cambridge Handbook of Creativity (pp. 447-463). Cambridge: Crambridge Press.

Krumm, G., Aranguren, M., Arán Filippetti, V., \& Lemos, V. (2016). Factor Structure of the Torrance Tests of Creative Thinking Verbal Form B in a Spanish-speaking Population. The Journal of Creative Behavior, 50(2), 150-164. https://doi.org/10.1002/jocb.76

Long, H. (2014). An empirical review of research methodologies and methods in creativity studies (20032012). Creativity Research Journal, 26(4), 427-438. https://doi.org/10.1080/10400419.2014.961781

López Martínez, O. \& Brufau, R. M. (2010). Creative intelligence and thinking styles. Anales de Psicología, 26(2), 254-258.

López Martínez, O. \& Navarro Lozano, J. (2008). Estudio comparativo entre medidas de creatividad: TTCT vs. CREA. Anales de psicología, 24(1), 138-142

Lubart, T. \& Besancon, M. (2017). On the measurement and mismeasurement of creativity (pp. 333-348). En R. Beghetto \& B. Sriraman (Eds.) Creative Contradictions in Education. Cham: Springer International Publishing.

Lubart, T. I., Zenasni, F., \& Barbot, B. (2013). Creative potential and its measurement. International Journal of Talent Development and Creativity, 1(2), 41-51.

Martinez Zaragoza, F. (2003). Características psicométricas del CREA. Un estudio con población española y argentina. Revista Iberoamericana de Evaluación Psicológica, 16(2), 71-83.

Park, M., Youngshin, M., \& Chun L. (2016). Revisiting individual creativity assessment: Triangulation in subjective and objective assessment methods. Creativity Reseach Journal, 28(1), 1-10. https://doi.org/10.1080/10400419.2016.1125259

Plucker, J. A., Beghetto, R. A., \& Dow, G. T. (2004). Why isn't creativity more important to educational psychologists? Potentials, pitfalls, and future directions in creativity research. Educational psychologist, 39(2), 83-96. https://doi.org/10.1207/s15326985ep3902_1

Primi, R., Nakano, T. D. C., Morais, M. D. F., Almeida, L. S., \& David, A. P. M. (2013). Factorial structure analysis of the Torrance Test with Portuguese students. Estudos de Psicologia (Campinas), 3O(1), 1928. https://doi.org/10.1590/S0103-166X2013000100003

Rinaudo, M. (2014). Estudios sobre los contextos de aprendizaje: arenas y fronteras. En P. Paoloni, M. Rinaudo \& C. González (Eds.), Cuestiones en Psicología Educacional. Perspectivas teóricas y metodológicas orientadas a la mejora de la práctica educativa (pp. 163-206). Cuadernos de Educación. La Laguna: Sociedad Latina de Comunicación Social.

Runco, M. (2010). Creativity: Theories and themes: Research, development, and practice. Boston: Elsevier Academic Press.

Runco, M. \& Acar, S. (2012) Divergent Thinking as an Indicator of Creative Potential. Creativity Research Journal, 24(1), 1-10. https://doi.org/10.1080/10400419.2012.652929

Runco, M., Abdulla, A., Paek, S., Al-Jasim, F., \& Alsuwaidi, H. (2016). Which test of divergent thinking is best? Creativity. Theories-Research-Applications, 3(1), 4-18.

Runco, M. \& Okuda, S. (1988). Problem-discovery, divergent thinking, and the creative process. Journal of Youth and Adolescence, 17, 211-220. https://doi.org/10.1007/BF01538162 
Silvia, P. J., Wigert, B., Reiter-Palmon, R., \& Kaufman, J. C. (2012). Assessing creativity with self-report scales: A review and empirical evaluation. Psychology of Aesthetics, Creativity, and the Arts, 6(1), 19-34. https://doi.org/10.1037/a0024071

Smith, J. \& Smith, L. (2017). The Nature of Creativity: Mayflies, Octopi, and the Best Bad Idea We Have. (pp. 21-35). En R. Beghetto \& B. Sriraman (Eds.), Creative Contradictions in Education. Cham: Springer International Publishing. https://doi.org/10.1007/978-3-319-21924-0_2

Sternberg, R. (2012). The assessment of creativity an investment-based approach. Creativity Research Journal, 24(1), 3-12. https://doi.org/10.1080/10400419.2012.652925

Sternberg, R. \& O'Hara, L. (2005). Creatividad e inteligencia. CIC. Cuadernos de Información y Comunicación, 10, $1-10$.

Torrance, E.P. (1990). The Torrance Tests of Creative Thinking: NormsTechnical manual figural (streamlined) forms $A \& B$. Bensenville: Scholastic Testing Service Inc.

Wolfradt, U. \& Pretz, J. (2001). Individual differences in creativity: personality, story writing and hobbies. European Journal of Personality, 15, 271-310. https://doi.org/10.1002/per.409

Yoon, C. H. (2017). A validation study of the Torrance Tests of Creative Thinking with a sample of Korean elementary school students. Thinking Skills and Creativity, 26, 38-50. https://doi.org/10.1016/j.tsc.2017.05.004

Zeng, L., Proctor, R. W., \& Salvendy, G. (2011). Can traditional divergent thinking tests be trusted in measuring and predicting real-world creativity? Creativity Research Journal, 23(1), 24-37. https://doi.org/10.1080/10400419.2011.545713

Recibido 30-08-2017 | Aceptado 14-02-2018

Este trabajo se encuentra bajo una Licencia Creative Commons Atribución 4.0 Internacional que permite a terceros utilizar lo publicado siempre que se dé el crédito pertinente a los autores y a Psicodebate 